\title{
MANTRA: Mobile Anticoagulant Therapy Management
}

\author{
Barbara Rita Barricelli, Yanet Devis, \\ Jose Abdelnour-Nocera, Jennie Wilson, John Moore \\ University of West London \\ London, UK \\ \{barbara.barricelli, jose.abdelnour-nocera, jennie.wilson, moorejo\}@uwl.ac.uk, ydevis@gmail.com
}

\begin{abstract}
The MANTRA (Mobile for ANticoagulant TheRApy) project is aimed at introducing mobile devices and innovative interaction design in anticoagulant therapy practice to improve the quality of life of patients and to support doctorpatient communication. This would have the benefit of saving both patient and healthcare workers time and resources and facilitate the monitoring of this therapy. The project is an ongoing usability study and proof of concept and this paper presents the results of a heuristic analysis performed to identify the potentials of this research.
\end{abstract}

Keywords-mHealth; mobile communication; self-testing devices; telemedicine; oral anticoagulant therapy

\section{INTRODUCTION}

Anticoagulant oral therapy is aimed at preventing the formation of thrombus. People at risk of developing thrombosis have to take anticoagulant treatment once a day in a dosage that varies depending on the results of an INR (International Normalised Ratio) test [1].

The two main reasons for coagulation therapy are venous thromboembolism and atrial fibrillation. Venous thromboembolism (VTE) [1][2] is the presence of coagulated blood, a thrombus, in a major blood vessel. VTE has the potential to cause significant harm by blocking blood flow to major organs and tissues. Atrial Fibrillation (AF) [3] is a cardiovascular disease that is associated with an increased risk of thrombus formation and risk of stroke in patients that are affected by it. The management of both VTE and AF requires treatment with Warfarin, an oral anticoagulation drug, in a dosage that needs to be adjusted on the basis of the INR test results. The INR test measures how long it takes for the blood to clot, i.e. the prothrombin time. The dose of anticoagulant is adjusted (increased or decreased) in order to maintain the INR result within an appropriate range. The INR is tested every 2-3 days on the commencement of treatment but once the levels have stabilised the interval between tests will change to between 7 and 20 days. INR tests are usually performed in clinics or laboratories therefore patients have to make frequent visits to have blood taken and for the doctor to make the decision about the appropriate anticoagulant dose. The patient must then take exactly the dose prescribed by the doctor. This can be difficult for elderly or disabled people and disruptive to their daily lives.

In this paper we present MANTRA (Mobile for ANticoagulant TheRApy), a project aimed at using mobile technology and innovative interaction design to improve the quality of life of patients with coagulation diseases. MANTRA proposes the introduction of digital solutions for the management of anticoagulation therapy in order to support remote doctor-patient communication. This would have the benefit of saving both patient and healthcare workers time and resources and facilitate the management of this therapy. The intention is to provide the patient with an INR self-testing device and a mobile device (an iPod) that transmits the INR result to the patients' doctor/clinic. The doctor is then able to review the result and transmit back to the patients the correct dose of anticoagulant that they need to take and when they need to repeat the INR test. In this setting, the goal is to provide patients with a testing device to be used at home to reduce the frequency of visits at the hospitals or clinics. Through the device and an iPod, the patients would be able to send their INR test results to their GPs and to receive back their prescriptions details. The focus of MANTRA Project is on studying the feasibility of using the proposed approach, and the patients' acceptability of using an interactive system. The project is a proof of concept and the objective is the development and evaluation of two interactive prototypes, one for doctors and one for patients. The usability evaluation is aimed at investigate the quality of the service offered by the applications and to understand how it may be improved.

In the next section, the state of the art of INR self-testing devices and INR tracking mobile applications is presented. Section III describes the MANTRA Project and its objectives. In section IV the evaluation of four mobile applications for iOS and Android is described and in the final section their results and the future developments are discussed.

\section{RESEARCH BACKGROUND}

\section{A. INR Self-Testing Devices}

INR self-testing devices are freely sold but not currently integrated into the systems for managing anticoagulant therapy adopted by the hospitals/clinics. 
INR self-testing devices allow to easily check the INR value with a finger stick test. Using a lancet, a drop of blood is placed on a test strip that is then inserted in the device. The devices uses three main methods to detect the INR value: 1) Monitoring of change in impedance of the sample when clotting occurs; 2) Mechanical endpoint clotting mechanism, monitored optically; and 3) Mechanical clot detection. With all the devices, the result is available within minutes.

INR devices can be used in two ways:

- Self-testing: the result has to be communicated to a doctor who is in charge of deciding the prescription of warfarin and the date of the next INR check.

- Self-monitoring: the patients, after a proper and adequate training, self-check their INR value and adjust their own warfarin dosage.

MANTRA Project is focused on self-testing approach, in that the aim is not to replace the fundamental role of the doctors in the anticoagulant therapy management, but to enable a remote communication among doctors and patients. Several studies (e.g., [3][4][5][6][7][8] report that the INR self-testing devices are reliable instruments and suitable alternative to conventional laboratory testing and that the INR results are typically very well reproducible and well correlated.

\section{B. INR Tracking Mobile Applications}

INR tracking mobile applications are available both for iOS (on iTunes) and for Android (on Google Play) mobile devices. They are applications aimed at personal tracking of one or more of these values: INR test results, medications, weight, and exercise. Some of them allow users to visualize charts of INR test results and medications dosage. None of them are designed to support doctor-patient communication. Some applications are available for free, while others may cost up to $£ 5$. In sections VI and VII, the results of the usability evaluations made on four applications (two for iOS and two for Android) are presented and discussed. None of the applications available on the iOS and Android stores are suitable for doctors. Therefore, as described in section IV, we performed the usability evaluations only from the patients' perspective.

\section{MANTRA PROJECT}

The MANTRA Project is focused on self-testing and aims at supporting the communication between doctor and patient in a remote setting, to allow the patients to manage their disease without affecting their freedom and improving the quality of their life. The research is qualitative, with no collection of blood samples or actual performance of INR tests. The information gathered from the interviews is related to the format of the interface and how it could be integrated into anticoagulation therapy monitoring, the pro and cons they identify in the application of such a proposal in their daily lives. The MANTRA Project is developed in the frame of a Leverhulme Trust Visiting Fellowship granted to the University of West London. The project started in January 2013 and is going to last one year. Given the limited time availability, the project's expected outcomes are more related to a proof of concept than to an actual evaluation of its concrete

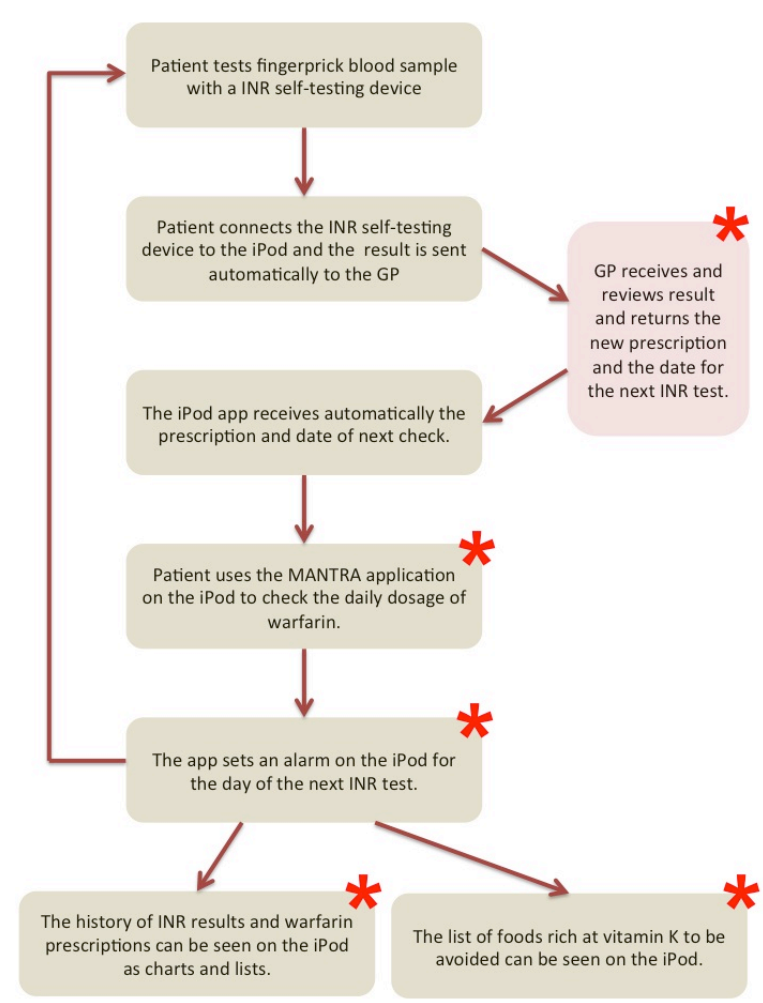

Fig. 1. The flow chart of the MANTRA scenario. The steps marked with a star are object of study and development of the MANTRA project.

application. However, we plan to develop two interactive prototypes, one for doctors and one for patients and to test them in the respective contexts of use with different usability evaluation methods. The MANTRA project focuses on studying: (a) The feasibility of using this approach, and (b) The patients' acceptability of using an interactive system. To meet aim (a), general practitioners (GPs) are going to be interviewed about their current processes for managing patients on anticoagulation therapy, the demographics of their patients receiving anti-coagulation, what factors would need to be considered in order to implement the digital solution and their views on its potential value. They are then going to be asked to participate in a further two face-to-face meetings to evaluate and then test the prototype digital interface. To meet aim (b), patients currently receiving anticoagulant therapy who volunteer to participate are recruited from anticoagulation clinics. They are going to be interviewed about their views and expectations of an interactive system for managing their therapy. In particular, we are going to explore patient requirements for the user interface and incorporate these into the design. They are then going to be asked to participate in a further two face-to-face meetings to evaluate and then test the usability of the prototype user interface. The phases of the MANTRA scenario are depicted in Fig. 1. The steps that are going to be considered in this year are marked with a red star.

\section{PRELIMINARY USABILITY EVALUATION}

We applied heuristic evaluation method [9] to test the usability of the four most used mobile applications available for iOS and Android. Two usability experts have been involved in the evaluations. For the evaluations we applied the 10 
Nielsen's heuristics: Visibility of system status, match between system and real world, user control and freedom, consistency and standards, error prevention, recognition rather than recall, flexibility and efficiency of use, aesthetic and minimalist design, help users recognize, diagnose, and recover from errors, help and documentation. We adopted Nielsen's severity ranking [10] to classify the usability issues: 1) Cosmetic problem only, 2) Minor usability problem, 3) Major usability problem, 4) Usability catastrophe. Heuristic evaluation is a socalled discount usability method that is especially useful in early stage of design. It is cheap and fast and does not require the participation of real end users. Since this evaluation was aimed at investigating the state of the art of the mobile applications for INR therapy management, and not at evaluating a result of the project, we preferred not to involve the patients at this stage. Patients, so as the doctors, will be later involved in usability evaluation of the prototypes we will design and develop.

\section{A. INR Tracking Mobile Applications and Usage Scenario}

We analysed two applications for iOS, "INR \& OAT Manager" [11] and "INR Pro" [12], and two for Android, "INR Tracker" [13] and "INR Tracker (Warfarin Log)" [14]. The two iOS applications allow to keep track of INR results and warfarin prescriptions. Both give the user the possibility to see the INR results history represented on charts. The two Android applications permit to track the INR results and warfarin prescriptions, but only "INR Tracker" offers the chart feature, while "INR Tracker (Warfarin Log)" allows to send an email with the INR result to addresses of user's choice. The scenario of use we defined for the evaluation is based on these tasks: 1) The user accesses the application; 2) The user saves the INR result; 3) The user browses the INR results history; 4) The user saves the warfarin prescription. These four tasks represent the most important features required by an INR tracker application and are all implemented in the four mobile applications under analysis.

\section{B. Personas}

Each of the two evaluators analysed each application twice using two different personas which profiles have been derived from interviews with doctors who have anticoagulant patients. The first persona is Emma. She is 52 years old and works as accountant in her husband's lawyer firm. Emma knows very well how to use a computer. She feels able to learn something new when it is just explained to her. If she needs IT help, she just searches the solution online. Because of her job, she regularly uses the Internet and iPad and iPhone. Emma uses her smartphone to make phone calls, to browse the web, to play games and uses many apps that she installed personally. Emma loves to travel but unfortunately she has been diagnosed with Pulmonary Thromboembolic Disease, and because of her condition, she has to make frequent visits to her GP to measure the INR values, so her trips cannot be as long as she desires. Emma dreams about a future where she no longer has to go to the doctor so often and where she can spend more time travelling and living a more relaxed life. The second persona is Rupert. He retired a year ago at the age of 64, after working for 30 years as train operator. Rupert is extremely active; he likes to go bowling once a week and loves his dancing classes. He is proud of his old car and spends an hour or two each day maintaining it in his garage. Rupert has being diagnosed with Proximal Atrial Fibrillation and since more than one year ago he has to take warfarin to manage his condition. His family is checking on him and forced him to carry an Android smartphone with him all the time. He only knows how to make calls and send SMS but does not use applications. Rupert's vision has gotten progressively worse over the past two years and he wears glasses most of the time. He uses the computer at home and accesses the Internet to email his older daughter who lives abroad. But when he cannot read because of the text size, he just gets frustrated and gives up. He desires to decrease doctors visits and to spend his time fixing his old car.

\section{Heuristic Analysis Results}

Table I shows the number of usability problems found by the evaluators impersonating Emma (E) and Rupert (R) personas. The problems are classified according to the severity ranking presented before in this paper.

\section{TABLE I. USABILITY PROBLEMS}

\begin{tabular}{|l|c|c|c|c|c|c|c|c|c|c|}
\hline & \multicolumn{10}{|c|}{ Number of Problems Classified on Severity Ranking } \\
\cline { 2 - 12 } & \multicolumn{1}{|c|}{ Cosmetic } & \multicolumn{2}{c|}{ Minor } & \multicolumn{2}{c|}{ Major } & \multicolumn{2}{c|}{ Catastr. } & \multicolumn{2}{c|}{ Total } \\
\cline { 2 - 11 } & $E$ & $R$ & $E$ & $R$ & $E$ & $R$ & $E$ & $R$ & $\boldsymbol{E}$ & $\boldsymbol{R}$ \\
\hline $\begin{array}{l}\text { INR \& OAT } \\
\text { Manager }\end{array}$ & 2 & 3 & 4 & 11 & 2 & 3 & 4 & 5 & $\mathbf{1 2}$ & $\mathbf{2 2}$ \\
\hline INR Pro & 4 & 2 & 5 & 2 & 4 & 2 & 5 & 7 & $\mathbf{1 8}$ & $\mathbf{1 3}$ \\
\hline INR Tracker & 1 & 2 & 4 & 3 & 1 & - & 2 & 2 & $\mathbf{8}$ & $\mathbf{7}$ \\
\hline $\begin{array}{l}\text { INR Tracker } \\
\text { (Warfarin) }\end{array}$ & 1 & - & 5 & 4 & 6 & 4 & 2 & 2 & $\mathbf{1 4}$ & $\mathbf{1 0}$ \\
\hline
\end{tabular}

The application with the highest number of problems (34) is "INR \& OAT Manager". The severity of the problems found by Rupert is so high that he may not even be able to perform the basic tasks requested. In particular, the registration of INR results and prescriptions are the two most affected tasks. For the other three applications, the evaluators have found more problems with Emma's persona. Emma represents the archetypal expert user of smartphones and mobile applications. Her familiarity with mobile interaction makes her keener to find errors and deficiencies in an application. Moreover, a user like Emma does not usually limit her exploration of an application to the main tasks but happens to be more interested in interacting with the application as a whole. On the other hand, Rupert being a novice in smartphone use is more oriented to keep the use of applications at minimum, because of his fear of the unknown. Table II shows the number of issues that may be resolved during use in time. In these cases, learnability dimension is respected and supports the user to become more aware of the applications potentials. However, the other usability issues deeply affects other usability dimensions, like effectiveness, use satisfaction and most of all, safety. Being the use of such applications related to health it is of great importance that safety, efficiency and effectiveness are respected. To better understand the kind of problems that affect the four mobile applications, we analysed them in respect with the 10 Nielsen principles. The four principles that appear to fail more are: Match between system and the real world, User 
control and freedom, Consistency and standards, and Error prevention.

TABLE II. LEARNABILITY

\begin{tabular}{|l|c|c|c|c|}
\hline \multirow{2}{*}{} & \multicolumn{2}{|c|}{$\begin{array}{c}\text { Usability } \\
\text { problems }\end{array}$} & \multicolumn{2}{c|}{$\begin{array}{c}\text { Resolvable in } \\
\text { time/use }\end{array}$} \\
\cline { 2 - 5 } & $E$ & $R$ & $E$ & $R$ \\
\hline INR \& OAT Manager & 12 & 22 & 8 & 9 \\
\hline INR Pro & 18 & 13 & 7 & 4 \\
\hline INR Tracker & 8 & 7 & 4 & 4 \\
\hline INR Tracker (Warfarin) & 14 & 10 & 6 & 6 \\
\hline
\end{tabular}

The problems that most seriously violate these four principles and that strongly affect effectiveness, efficiency and safety use of the applications may be classified in three main classes: Flexibility, readability, and data representation. The applications show three serious issues in terms of flexibility that have been spotted by all the evaluations, both with Emma and Rupert personas. The first issue regards the possibility of registering INR test results using a date that is different from the current one. This may represent a limitation for the patient that may be interested in registering results of tests taken in the previous days. Without this feature the patients are forced to register the INR test results with wrong dates putting at risk their own safety. A second problem is the unit used to specify the dosage of warfarin that the doctor prescribes. One of the applications asks the patient to set a default unit measure (mgs of warfarin contained in a tablet). After this setting operation, all the prescription registrations done with the application are expressed in term of "units" (e.g. tablets) and no more in "mg". This may represent a serious problem because of the different format of warfarin tablets sold around the world. In the UK and several other countries, warfarin tablets are distributed in different blister packages, each one having different mgs of warfarin, with tablets of different colours (one colour for each warfarin dosage). This implies that the patients may need to take each day tablets of different kind (colour/dosage) and therefore a default "unit" is not suitable for them. Besides, in other European countries, like Italy, and in other countries, like Venezuela and Colombia, warfarin is distributed in tablets that may be cut into parts to obtain different dosages of the medicine. Also in this case, the default "unit" is not the correct solution for the patients. The last issue of this class of problems regards the interval of prescription: most of the applications do not allow the patients to freely specify the initial and final date of the prescription received by their GP. Typically, the applications offer the possibility to register a daily prescription (registered day by day) or a weekly prescription (from Monday to Sunday, with no possibility of personalizing this format). As to the readability class of problem, the evaluations made with Rupert's persona pointed out the great importance of using fonts, colours (for text and background) and icons that may be easily read by patients. One important option could be to implement a feature for resizing the text size to support the accessibility especially for elderly and/or patients with sight problems. The issues related to the data representation class regard the possibility of browsing the INR test results history of the patient. It is of fundamental importance for the patients to be able to access the previous tests results to be more aware of their health conditions. To this aim, it is of great importance to offer a feature for INR test results representation in charts and lists. Some of the applications already offer such charts and lists but they are not enough flexible and interactive. For example, it is not possible to specify a range of dates to use for the data visualization and the charts/lists are not interactive, i.e. the values visualized are not clickable and is not possible to see more information about the INR values and relative prescriptions.

\section{CONCLUSIONS AND FUTURE DEVELOPMENTS}

Despite the limits of the heuristic evaluation method, this preliminary analysis allowed us to understand which are the potentials of the use of mobile devices in this context. On the basis of the results be obtained by this preliminary usability evaluation on the existing mobile applications for INR tracking, we plan to design and realize a low-fidelity prototype of the user interfaces we need to develop for patients and doctors. The prototypes will be tested with patients and doctors and the results of the tests will be used to proceed with the design and development of a first version of the user interfaces for iOS operating system.

\section{REFERENCES}

[1] A.T. Cohen, G. Agnelli, F.A. Anderson, J.I. Arcelus, D. Bergqvist, J.G. Brecht, I.A. Greer, J.A. Heit, J.L. Hutchinson, A.K. Kakkar, D. Mottier, E. Oger, M. Samama, M. Spannagl, "Venous thromboembolism (VTE) in Europe - The number of VTE events and associated morbidity and mortality," Thromb Haemost, vol. 98, pp. 756-764, 2007.

[2] C. Kearon, E.A. Akl, A.J. Comerota, P. Prandoni, H. Bounameaux, S.Z. Goldhaber, M.E. Nelson, P.S. Wells, M.K. Gould, F. Dentali, M. Crowther, S.R. Kahn, "Antithrombotic Therapy For Vte Disease," CHEST, vol. 141(2_suppl), pp. e419S-e494S, 2012.

[3] S. Schneeweiss, J.J. Gagne, A.R. Patrick, N.K. Choudhry, J. Avorn, "Comparative Efficacy and Safety of New Oral Anticoagulants in Patients With Atrial Fibrillation," Circulation: Cardiovascular Quality and Outcomes, vol. 5, pp. 480-486, 2012.

[4] H.I. Bussey, E. Chiquette, T.M. Bianco, K. Lowder-Bender, M.A. Kraynak, W.D. Linn, L. Farnett, G.M. Clark, "A statistical and clinical evaluation of fingerstick and routine laboratory prothrombin time measurements," Pharmacotherapy, vol. 17(5), pp. 861-866, 1997.

[5] M.E. Cromheecke, M. Levi, L.P. Colly, B.J.-M. de Mol, M.H. Prins, B.A. Hutten, R. Mak, K.C.J. Keyzers, H.R. Büller, "Oral anticoagulation self-management and management by a specialist anticoagulation clinic: a randomised cross-over comparison," The Lancet, vol. 356(9224), pp. 97-102, 2000.

[6] D.M. Dorfman, E.M. Goonan, M.K. Boutilier, P. Jarolim, M. Tanasijevica, S.Z. Goldhaber, "Point-of-care (POC) versus central laboratory instrumentation for monitoring oral anticoagulation," Vascular Medicine, vol. 10(1), pp. 23-27, 2005.

[7] C. Gardiner, K. Williams, I.J. Mackie, S.J. Machin, H. Cohen, "Patient self-testing is a reliable and acceptable alternative to laboratory INR monitoring," Br J Haematol, vol. 128(2), pp. 242-247, 2005.

[8] A. Tripodi, "Prothrombin time international normalized ratio monitoring by self-testing," Curr Opin Hematol, vol. 11(3), pp. 141-145, 2004.

[9] J. Nielsen, R. Molich, "Heuristic evaluation of user interfaces," Proc. ACM CHI'90 Conf., pp. 249-256, 1990.

[10] J. Nielsen, R. Mack (Eds.), Usability inspection methods, Wiley, New York (1994)

[11] INR \& OAT Manager. http://bit.ly/10ELb65

[12] INR Pro. http://bit.ly/16Mqaa7

[13] INR Tracker. http://bit.ly/10Q0iGT

[14] INR Tracker (Warfarin Log). http://bit.ly/10Ju95h 